\title{
The Zoomorphic Miro Robot's Affective Expression Design and Perceived Appearance
}

\author{
Moojan Ghafurian ${ }^{1}$ (D) $\cdot$ Gabriella Lakatos ${ }^{2} \cdot$ Kerstin Dautenhahn $^{3}$
}

Accepted: 10 September 2021 / Published online: 4 January 2022

(c) The Author(s), under exclusive licence to Springer Nature B.V. 2021

\begin{abstract}
This article proposes design guidelines for 11 affective expressions for the Miro robot, and evaluates the expressions through an online video study with 116 participants. All expressions were recognized significantly above the chance level. For six of the expressions, the correct response was selected significantly more than the others, while more than one emotion was associated to some other expressions. Design decisions and the robot's limitations that led to selecting other expressions, along with the correct expression, are discussed. We also investigated how participants' abilities to recognize human and animal emotions, their tendency to anthropomorphize, and their familiarity with and attitudes towards animals and pets might have influenced the recognition of the robot's affective expressions. Results show significant impact of human emotion recognition, difficulty in understanding animal emotions, and anthropomorphism tendency on recognition of Robot's expressions. We did not find such effects regarding familiarity with/attitudes towards animals/pets in terms of how they influenced participants' recognition of the designed affective expressions. We further studied how the robot is perceived in general and showed that it is mostly perceived to be gender neutral, and, while it is often associated with a dog or a rabbit, it can also be perceived as a variety of other animals.
\end{abstract}

Keywords Affective expression $\cdot$ Emotion $\cdot$ Zoomorphic robot $\cdot$ Social robot $\cdot$ Miro $\cdot$ Emotionally intelligent robot

\section{Introduction}

Affective expressions are one of the effective communication channels among humans and many other animals, which can significantly affect quality of interactions. This commu-

This research was funded by the Network for Aging Research at the University of Waterloo. This research was undertaken, in part, thanks to funding from the Canada 150 Research Chairs Program.

$凶$ Moojan Ghafurian

moojan@uwaterloo.ca

Gabriella Lakatos

g.lakatos@herts.ac.uk

Kerstin Dautenhahn

kerstin.dautenhahn@uwaterloo.ca

1 Department of Electrical and Computer Engineering, University of Waterloo, Waterloo, ON, Canada

2 Adaptive Systems Research Group, University of Hertfordshire, Hatfield, UK

3 Departments of Electrical and Computer Engineering, and Systems Design Engineering, University of Waterloo, Waterloo, ON, Canada nication approach has also attracted interest in robotics and virtual agent research, in particular when the robots or agents directly interact with humans. In fact, along with other factors such as responsiveness to social cues, social exchange [1], and social content [2], the capability to express emotions is believed to highly affect and improve the quality of humanrobot interaction. It has been confirmed by multiple studies that affective interaction in intelligent agents can increase users' engagement [3], enjoyment, and cooperation with the technology [4,5].

There are many challenges in implementing emotions in virtual agents and social robots. While understanding emotions, especially basic emotions, is considered to be intuitive for humans [6], both children and adults have difficulty in distinguishing between some emotions in human images, such as anger and disgust $[7,8]$. Further, limitations in movements of social robots or in the design of virtual agents can affect how affective expressions are implemented.

In this paper, we study how to make the Miro robot-a biomimetic animal-like robot $[9,10]$ —emotionally expressive. Miro has been designed as a social robot with a zoomorphic embodiment, but does not mimic any specific 
animal, and is capable of communicating through multiple communication methods such as body language [9]. Adding emotional expressions to Miro is an important step towards making it socially and emotionally intelligent, as well as improving multi-modal interactions with Miro. While a study by Collins et al. (2015) [11] has provided an initial design and pilot evaluation for emotions of Miro reflected through its LEDs, to the best of our knowledge, ours is the first work towards implementing emotional expressions on Miro, expressed by its facial and body gestures.

In the following, we will provide the design and evaluation of a set of eleven complex and simple affective expressions for Miro. This set includes both emotions (e.g., excited, sad) and moods (e.g., tired, bored). Those were selected since they could be beneficial in a number of applications where people might benefit from interactions with a social robot such as Miro, for example in dementia care, for supporting older adults, or for therapy and education for children.

The article is structured as follows. We will first introduce the related work and then describe the design for Miro's affective expressions. Afterwards, we will present a crowdsourcing video study, evaluating the designed affective expressions on Amazon Mechanical Turk. We will conclude by providing a discussion of the results and pointing out the limitations of this work.

\section{Background}

Emotional expressions of robots and Virtual Agents (VAs) have been studied widely [11-23]. While most studies focused on facial expressions in the context of human-like or android robotic, or virtual agents, there are also studies that used different modalities, such as using lights, sounds, motion, and body gestures to convey emotions (e.g., see [22]). Not having to rely on agents with a face that can generate human-like affective expressions is particularly attractive for either machine-like, 'appearance-constrained' robots that lack particular human-like features [24], or zoomorphic robots.

Methodologically, one of the common approaches for evaluating the emotions of robots and VAs is using video studies. For instance, Breazeal (2002) [19] designed and evaluated nine emotional expressions for Kismet, one of the first emotionally expressive, interactive robots, having face action units similar to humans. Evaluations were done through studies with images, followed by video studies of 7 of the expressions. Most of the expressions had a higher accuracy of recognition in the video study, as opposed to the still images. More recently, Korcsok et al (2018) used a video study to test six emotional displays of an abstract agent (five emotions and one neutral state), which was inspired by biological and ethological rules. They showed that while par- ticipants were able to successfully recognize most emotions (with anger being the most easily recognizable emotion), fear and sadness were the emotions that were most often confused [20]. Furthermore, Gacsi et al. (2016) used a video study to evaluate emotional behaviours of the non-humanoid PeopleBot robot. Five emotions for the robot (fear, anger, joy, and neutral) were inspired by ethological findings on dog behaviour, and the study included emotional displays of actual dogs. Gacsi et al. (2016) showed that the participants successfully understood both the robot's and the dog's emotional states that were shown in the videos [21].

In another study, 13 Action Units (AUs) were used to change facial actuators of the cartoon-like Probo robot, resulting in the design of a set of eight emotions. $88 \%$ accuracy in recognition of emotions was achieved for a virtual model of the robot, compared to $83-84 \%$ for the physical robot. The study discussed that a virtual model may be better controllable than the robot itself which might explain higher recognition rates [18]. Further, Numata et al. (2019) implemented eight emotions (with three variations for each) for a non-human virtual agent (Piyota) that looked like a chick, and argued that older adults show a higher degree of variability in recognition of the emotions as compared with younger adults [17].

An initial attempt towards making the Miro robot emotionally intelligent was reported in [11], using six RGB LEDs located on Miro's sides. Depending on the specific emotion, the LEDs changed in (a) color (red, white, and green), (b) intensity of the color (from zero to actual intensity), and (c) rate of color changes (slow, medium, and fast). Color was used to show valence of emotion, and the rate of light changes represented arousal. A pilot study with five participants using a robot simulator suggested that these patterns of pulsating lights can be effective in representing valence and arousal of emotions [11]. However, using LEDs as a modality for affective expressions has its limitations. For example, recognition of LED lights might be affected by users' conditions such as color-blindness, or their selective attention to or preference for certain colors. Furthermore Miro's LEDs may not be noticeable from specific angles, in a room that is well-lit, or in outdoor applications (e.g., with bright sunlight).

While the design of affective expressions for many robots, and especially virtual agents, were inspired by humans' emotions, inspiration from the behaviour of animals might be more effective for animal-like robots. To that end, dog behaviour has attracted attention by researchers, as (a) there is a considerable amount of studies on dog behaviour, which can be also implemented in robots [25], (b) understanding some of dogs' reactions can be intuitive for humans (e.g., dog owners are shown to be capable of understanding different emotions in dogs, such as joy, jealousy, curiosity, fear, and sadness [26,27]), and (c) dogs are capable of developing 
effective social interactions with humans despite their less complex cognitive capacities [28].

Given that people tend to treat and interact with robots of a zoomorphic appearance similar to how they would treat the animal that the robot is modelled after [29], it is plausible to expect that understanding a zoomorphic robot's affective states might be influenced by similar factors that affect humans' understanding of animal emotions. Many factors such as age, gender, and pet ownership have been studied to investigate people's understanding of animal emotions. For instance, Dawson et al. [30] provided evidence that gender and age can affect accuracy of recognition of cat emotions, with pet ownership having only a very small positive impact. Further, the effect of pet ownership on recognition of animal emotions has not been consistent across the literature [30]. For example, while some studies emphasized that experience can significantly affect recognition of animals' emotions [31,32], others observed only little improvements $[30,33]$. Likewise, people with varying levels of experience with dogs, being asked to describe the emotional content of dog barks, achieved similar scores [34]. Tami and Gallagher [35] suggested that without a theoretical background, having experience with dogs (e.g., being a dog owner) may not improve recognition of dog emotions. Further, Demirbas et al. [36] provided evidence that dog owners may even perform significantly worse when classifying dogs' emotional states in a child-dog interaction task. Thus, while the animal literature does not provide a clear relationship between pet ownership and recognition of animal emotions, investigating the different, but related impact of pet ownership and familiarity with animals on recognition of a zoomorphic robot's affective states, as relevant to the present study, emerges as an interesting direction of investigation.

Lastly, while the existing studies, specially those on VAs, have used a range of different emotions, the emotion set used in studies on social robots are usually limited (e.g., restricted to the subset of basic emotions, perhaps because both machines and humans are better in recognizing them [37]). But a larger set is more beneficial, as it can enrich social interactions with robots and increase humans' engagement with them [37]. To this end, in the present study, we investigated the feasibility of designing a larger set of 11 effective expressions, including, but not limited to the basic emotions.

\section{Affective Expressions for the Miro robot}

\subsection{Artifact: The Miro robot}

The Miro robot is a commercially available robot designed by the company Consequential Robotics as an animal-like robot companion. It is aimed at application areas including being a companion robot and assistant for older adults, a tool in robot- assisted therapy, or being used in education and teaching. ${ }^{1}$ It is a fully programmable, ROS enabled, autonomous robot. Its sensors include stereo vision, ultrasonic sensors, light level, infrared cliff sensors, as well as tactile sensors on its body and head. In this study we focused on using its range of motion and expressive abilities, including tail wagging and drooping, blinking, ear-rotation, head movements, and colored lights (LEDs). It can move around on smooth surfaces. Its head movements are particularly expressive: It has three Degrees of Freedom (DoF) in the neck providing lift, pitch, and yaw rotations. Additional DoFs include rotation for each ear, tail droop and wag, and eyelid open/close.

\subsection{Design of Affective Expressions}

As mentioned above, Miro is a zoomorphic (animal-like) robot that was intentionally designed in a way that it does not look like any specific animal. However, it has similarities to a dog. As dog behaviour is well documented and studied, we took inspiration from the literature on dog behaviour (cf. [21]) for the design of Miro's affective expressions, especially for those emotions that are studied extensively for dogs (i.e., happy, sad, fearful, and angry). We used behaviours such as (a) the feeling of sadness caused by being separated from the owner [39], (b) fearful [40] and angry [41] because of a threatening stranger, and (c) happy to see an owner and greeting him/her $[39,42]$.

Designing more complex emotions was more challenging for two reasons: (a) while dog behaviour has been studied extensively, we could not find detailed descriptions of some more complex emotional expressions (e.g., disgust), and (b) Miro's range of movements is much more limited than dogs' due to multiple constraints of its design which, e.g., prohibits bending of the body and ears. Therefore, we also considered alternative sources for the design of affective expressions, e.g., research on the behaviour of other mammal species (rabbits [43], mice [44], and humans), as well as cartoon characters.

Tail movements, shapes of ears, and shapes of muzzles are three aspects that have seen a lot of attention in the literature on animal emotions. Here, we designed Miro's tail movements based on the literature on dogs' tail movements $[45,46]$. However, due to the limitations, the robot's range of movements is much more limited as compared with dogs. Also, the shapes of ears and muzzles cannot be controlled in Miro and Miro's ears cannot be bent, which are important for reflecting some emotions in animals. However, the robot's ears can be rotated inwards and outwards.

As Miro's shape and size of the ears are very similar to a rabbit's ears, we referred to the literature on the behaviour of rabbits [47] to specify the angle of ears for each emotion,

\footnotetext{
${ }^{1}$ URL: http://consequentialrobotics.com/, last accessed 21 July 2020.
} 


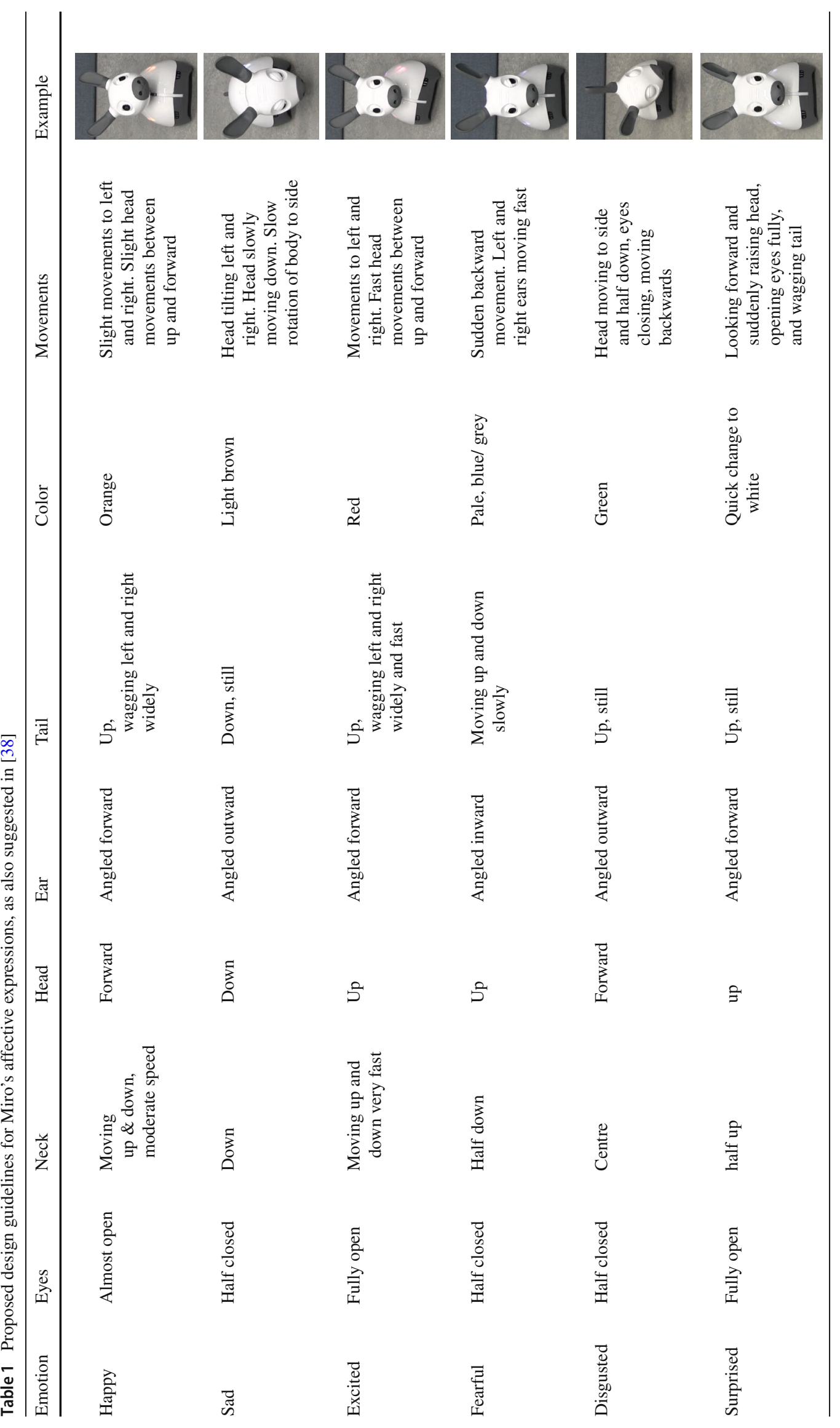




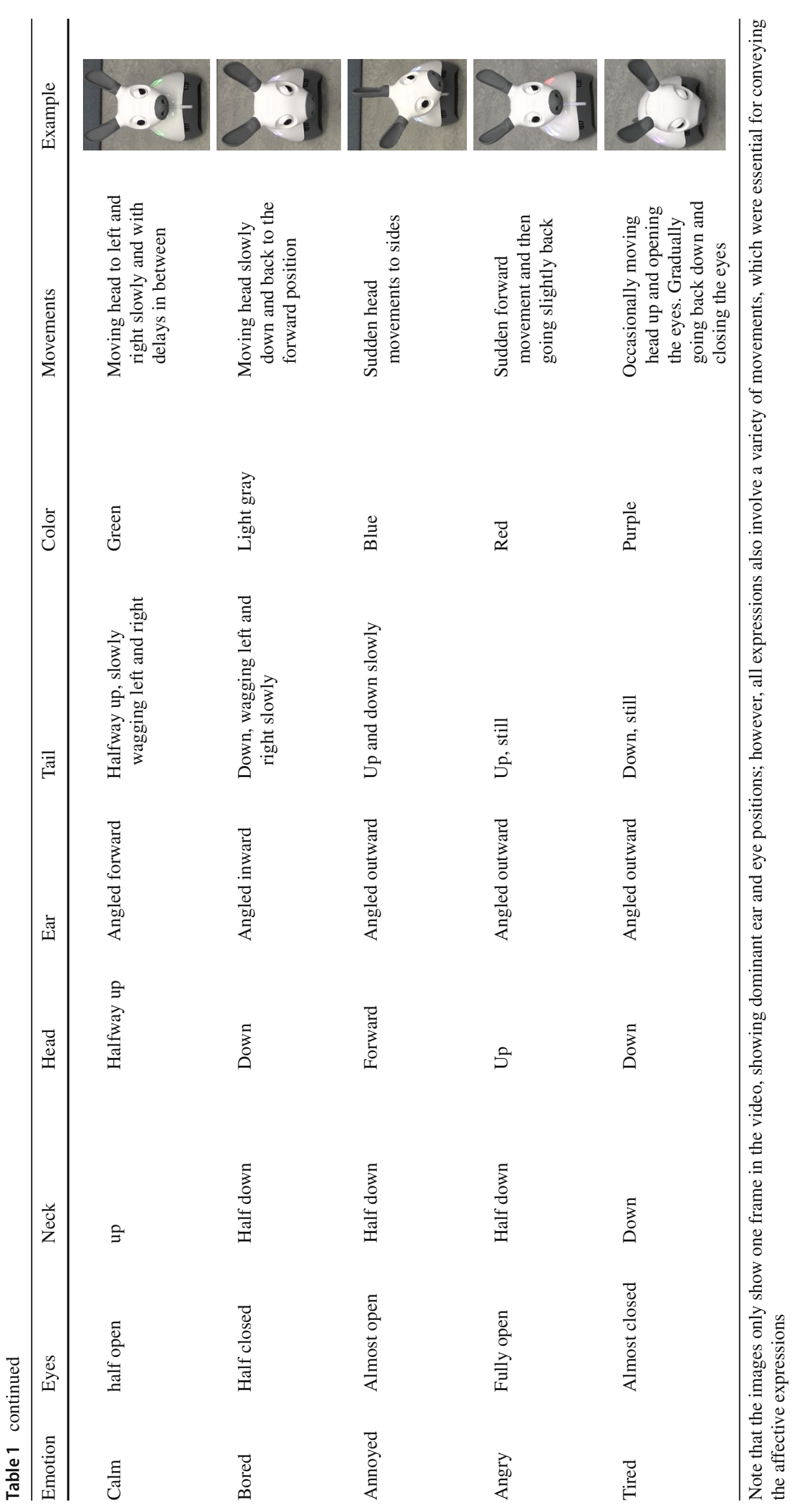


while we also referred to the literature on mice behaviour, which describes ear rotation in detail [44].

Furthermore, for body movements, along with inspiration from animal behaviour (e.g., being mostly inactive and looking drowsy when animals are bored [48]), we referred to humans (e.g., moving backwards when being afraid) and cartoon characters (e.g., moving the head away when disgusted). Also, the design of the robot's movements of the eye lids were mostly based on humans (e.g., wide eyes for surprise [49]), as we assumed that it could be more intuitively understood by humans. Lastly, color was decided according to the existing work on the mapping of emotions to colors [50,51]

Table 1 shows the final design, as well as an image illustrating each affective expression. Note that an image alone may not be representative of the behaviour, as body and head movements play an important role in showing Miro's affective expressions, which cannot be captured by the images.

\section{Experiment}

The experiment was conducted as a video study on Amazon Mechanical Turk and addressed the following research questions.

- RQ1 How do participants map Miro robot's affective behaviour to the designed affective states shown in the videos? Here, we were interested in participants' choices as well as their justifications for these choices.

- RQ2 Do participants' abilities to recognize human and animal emotions have an impact on their recognition of the robot's designed affective expressions? We expected that the better participants are in recognizing humans' and animals' emotions, the higher their accuracy in recognizing the robot's affective expressions will be.

- RQ3 How do participants' tendencies to anthropomorphize affect participants' recognition of Miro's expressions? We hypothesized that the attribution of human-like characteristics might interfere with participants' recognition of the robot's expressions (as it is shown to affect participants' behaviour towards intelligent agents [52]), since the robot's expressions were predominantly modelled based on animal behavior.

- RQ4 Does participants' familiarity with, exposure to, or attitudes towards animals impact their recognition of the designed affective expressions of Miro? We tentatively expected that people who either have or had pets, or are very familiar with them, might be better in recognizing those expressions, although, as discussed above, the literature in this regard is inconclusive. Understanding the possibility of these effects would also help us with elimi- nating any possible confound when studying RQ1, RQ2, and RQ3.

- RQ5 This question concerned the general perception of Miro and consisted of three sub-questions: Which gender do participants attribute to Miro? Which animal does it remind them of? How do participants perceive the nature of the robot, machine-like, human-like, animallike? These were exploratory questions that we were interested to answer, since we can expect that in many future application areas those aspects will play a role in the suitability of the robot for certain tasks (e.g., as these aspects can also affect users' perception of the robot [53]), as well as expectations that users might have of the robot's capabilities, which will impact its acceptance by users. Since Miro was created as a zoomorphic robot, and we designed its affective expressions largely based on animal behaviour, we expected that participants would not have a strong human-like view of the robot.

\subsection{Method}

The study had three parts: evaluation of Miro's expressions, questionnaires, and evaluation of human emotions from images. These parts are described in the following sections.

\subsubsection{Evaluation of Miro's Affective Expressions}

In this part, participants watched and evaluated the 11 affective expressions. The videos were shown in a random order and we asked the participants to decide on the emotion/affective state shown in the video. The videos were taken from two different angles (see Fig. 1). One from front (so that the participants can see Miro's head and face properly), and one from the side (e.g. to show tail movements). During filming, two cameras were used to take the videos simultaneously and both cameras were fixed at the exact same height (about $120 \mathrm{~cm}$ from the ground) in a way that it represents a realistic view of Miro from the perspective of a person who might interact with it. Positions of the cameras and Miro were kept constant during the filming of all affective expressions. ${ }^{2}$

After watching each video, participants were asked (1) whether they were able to see the video (in order to check for possible software problems), and (2) to select what emotion they thought Miro displayed in the video. Participants were given an additional, "not sure", choice. However, they had to select another option along with "not sure", as we wanted to know what their best guess was, even if they were not sure about their response. Participants could select as many

\footnotetext{
2 The videos and the underlying code will be shared upon request to allow other researchers to either replicate the study or use the expressions in their own research projects.
} 


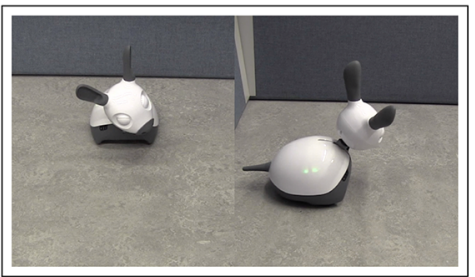

(a)

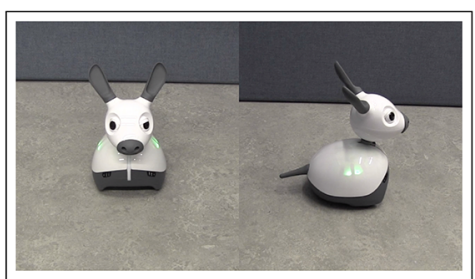

(b)

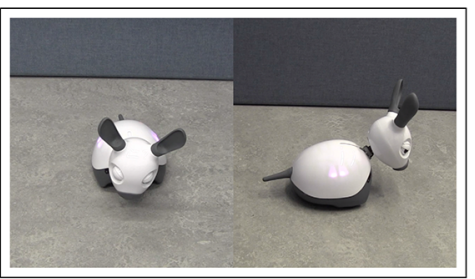

(c)

Fig. 1 Example of the two camera angles for a Disgust, $\mathbf{b}$ Calm, and $\mathbf{c}$ Tired

emotions as they thought might be representative of what they saw in the video and were able to re-play the videos as many times as they wished. The questions were only displayed after each video was played to the end.

To understand deviations from the hypothesized response and to get feedback that can help with improving the designs, participants were asked to explain their choice when it was different from the expression in the video that we had designed originally. Participants were told that they will be asked to explain their choice "randomly" during the study. We did not request participants to explain their choices for each video they watched, in order to avoid fatigue. Participants could also watch the video again and re-play it multiple times before explaining their choice, however, they could not change their original responses.

\subsubsection{Questionnaires}

Three questionnaires were used to gather information about participants' (a) demographics, (b) previous exposure and experience with animals, and (c) tendency to anthropomorphize. These questionnaires are described below.

(a) Demographic Questionnaire gathered participants' age, gender, level of education, and culture/ethnicity.

(b) Animal Exposure Questionnaire asked (1) whether the participants have ever had a pet (and if yes, what their pet was), (2) whether they have a close friend/family member who has a pet (and if yes, what their pet was). They were also asked general questions about their attitude towards pets, such as (3) whether they liked pets, (4) if they were scared of pets, (5) whether they have difficulty in understanding pets' emotions (as well as a consistency check by asking if they had a good understanding of pets' emotions), and (6) whether they believed that animals can have emotions like humans. Finally the participants were asked to indicate how (7) machine-like, (8) animal-like, and (9) human-like Miro was.

(c) IDAQ Questionnaire proposed by Waytz et al. [54] was used to measure participants' tendency to anthropomorphize, which we hypothesized could affect participants' perception of Miro.
Additional questions were included in the Animal Exposure and IDAQ questionnaires as attention checks.

Furthermore, to understand how the participants perceived Miro's gender and the animal it most reminds them of, we asked (1) what animal they thought Miro was, and (2) what Miro's gender was in their opinion.

\subsubsection{Human Emotion Understanding}

To study participants' understanding of emotions in general, we asked them to evaluate 12 images of humans, which showed six different emotions: anger, fear, sad, surprise, happy, and neutral (the FacesDB ${ }^{3}$ dataset was used, from which we used a subset of the 11 expressions for Miro). For each emotion, we had two pictures, where skin color, gender, and age of the people shown were counterbalanced (e.g., a young female and an old male for the 'surprise' emotion).

\subsection{Procedure}

Participants first accepted the consent form and read the instructions. Afterwards, they watched a general video, which combined all different affective expressions (in order for participants to get familiar with Miro and its range of movements). Afterwards, participants viewed the videos showing different affective expressions and related questions one by one, in a random order. Next, participants were asked to complete the questionnaires. Finally, they viewed the images of human expressions (in the same order for all participants) and evaluated them.

\subsection{Participants}

147 participants completed the task on Amazon Mechanical Turk. Participation was limited to people based in the USA and Canada, and MTurk Workers who had completed at least 50 HITs and had an approval rate of over $96 \%$. The data from 31 participants were removed as they failed to pass the attention and sanity checks. This left data from 116 participants ( 41 female, 71 male, 4 gender unknown; age range

\footnotetext{
${ }^{3}$ http://app.visgraf.impa.br/database/faces/.
} 


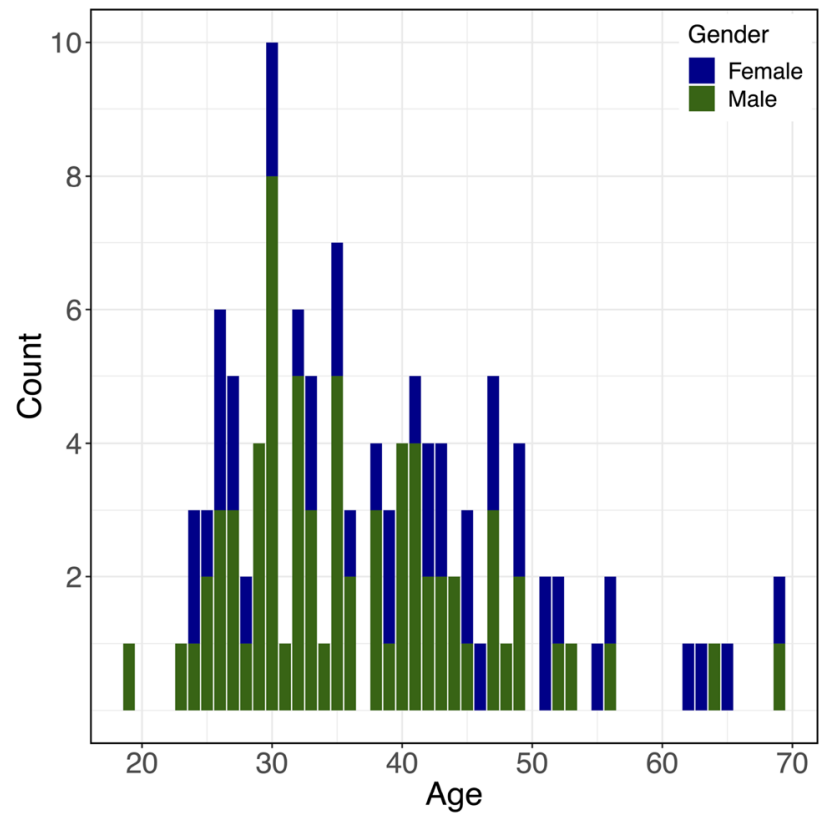

Fig. 2 Participants' age distribution

19-69 years, average: 38 years). Figure 2 shows participants' age distribution. In internal test trials we estimated that the study would take about 40 minutes. Participants were paid 4 USD for completing the study and a pro-rated amount if they wished to stop earlier. The study received full Ethics clearance from the University of Waterloo's Research Ethics Committees.

\section{Results}

We first present the results on the evaluation of Miro's affective expressions, followed by the human emotion recognition results and the results of the questionnaires.

\subsection{RQ1: Evaluation of the Robot's Designed Affective Expressions}

Table 2 and Fig. 3 show the results of evaluations of Miro's affective expressions. The correct response was selected significantly higher than random for all expressions $(p<.0001$ for all, measured using binomial tests). Also, in all expressions except "Fearful" and "Bored", the correct option was selected more often than the others. This difference was significant (calculated through binomial tests) for "Happy" $(p<.001)$, "Sad" $(p<.001)$, "Excited" $(p<.05)$, "Surprised" ( $p<.001)$, "Calm" $(p<.01)$, and "Tired" $(p<.001)$. For the others, there were more than one option (the correct option) that were similarly selected significantly more than random. To understand what aspects of the design

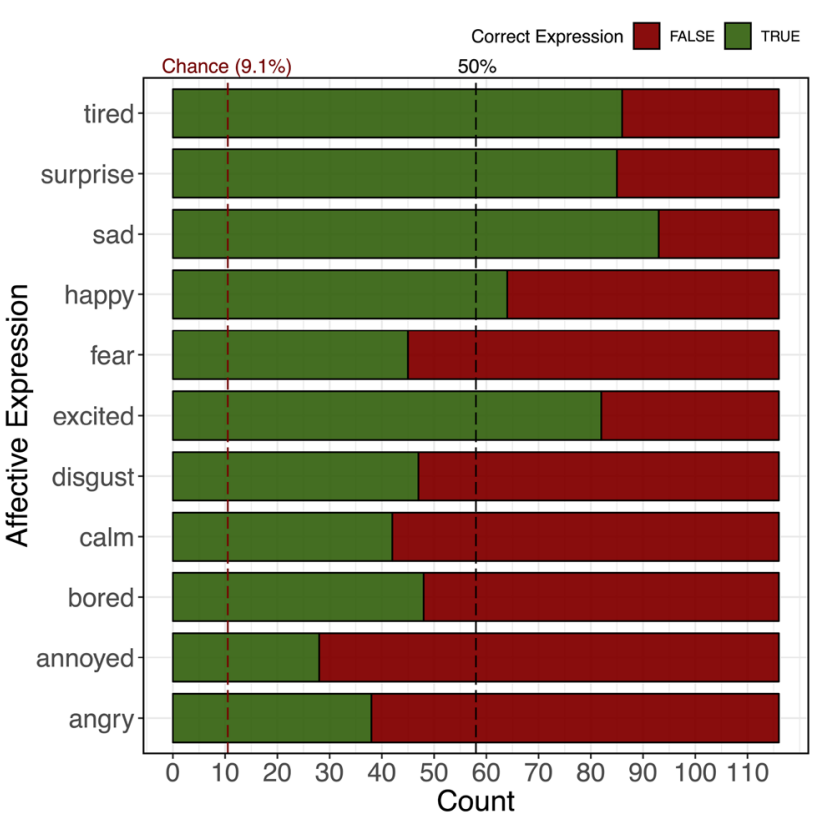

Fig. 3 Evaluation of the designed 11 affective expressions. The correct answer was selected significantly above the chance level for all the expressions $(p<.0001$ in all $)$

led to confusing some expressions with others, we studied participants' comments:

Bored was mostly confused with "Tired" and "Sad". In most cases, this was due to participants paying attention to the features that "Bore" has in common with the other two expressions, especially in eyes (e.g., eyes being half open) and the position of the head. Below are some examples of participants' comments.

- "I thought Miro was tired because he looked like he was fighting to keep his eyes open and his head up. Both these things happen to me when I get tired."

- "I thought Miro was tired because the way Miro kept his eyes semi closed."

- "I thought Miro was tired because of his drooping eyelids and slowing tail wag. His head also drooped."

- "Miro's eyes shut half-way, head tilts down - could be sad or tired."

- "Miro looks like it is feeling depressed. Its eyes are half closed."

- "He seems to be either sad or tired, slowly dropping his head and his eyes are not fully open."

Fearful was mostly confused with "Surprised". This was again due to the similarities in eye and body movements between the two expressions. While not selected, "Fearful" can be inferred from some of the comments. Below are some examples. 
Table 2 Correct expressions are shown in bold

\begin{tabular}{llllllllllll}
\hline & Happy & Sad & Excited & Fearful & Angry & Disgusted & Surprised & Calm & Bored & Annoyed & Tired \\
\hline Happy & $\mathbf{6 4}^{* * *}$ & 2 & 27 & 10 & 1 & 1 & 10 & $\mathbf{3 0}$ & 5 & 5 & 2 \\
Sad & 1 & $\mathbf{9 3}^{* * *}$ & 1 & 5 & 2 & 6 & 0 & 2 & 8 & 3 & 29 \\
Excited & $\mathbf{5 9}$ & 1 & $\mathbf{8 2}^{*}$ & 9 & 3 & 0 & 3 & 4 & 1 & 3 & 2 \\
Fearful & 9 & 1 & 19 & $\mathbf{4 5}$ & 7 & 2 & 46 & 3 & 3 & 5 & 2 \\
Angry & 12 & 1 & $\mathbf{2 0}$ & 9 & $\mathbf{3 8}$ & 5 & 5 & 6 & 13 & $\mathbf{2 7}$ & 1 \\
Disgusted & 4 & 13 & 3 & $\mathbf{4 2}$ & 11 & $\mathbf{4 7}$ & 5 & 4 & 3 & $\mathbf{2 3}$ \\
Surprised & 7 & 6 & 17 & 7 & 5 & 0 & $\mathbf{8 5}^{* * *}$ & 4 & 3 & 5 \\
Calm & 4 & 9 & 4 & 12 & 17 & 10 & 6 & $\mathbf{4 2}$ & 14 & 19 & 12 \\
Bored & 1 & $\mathbf{3 2}$ & 2 & 2 & 3 & 0 & 3 & 10 & $\mathbf{4 8}$ & 5 \\
Annoyed & 15 & 1 & 14 & 5 & 6 & 8 & 19 & 13 & 17 & $\mathbf{2 8}$ & 54 \\
Tired & 3 & $\mathbf{3 3}$ & 2 & 3 & 0 & 3 & 0 & 3 & 10 & 1 \\
\hline
\end{tabular}

Bolditalic shows the expressions that were mostly confused with the original, designed expressions (i.e. they received more than 20 votes). Rows show the actual expressions and columns show participants' selections. Options that were selected more than the correct expression are shown in italic. Through binomial tests, we show if the correct emotion was selected significantly more than the other options: ${ }^{* * *}: p<.001{ }^{* * *}: p<.01$, $*: p<.05$

- "I thought Miro was surprised because they kind of jumped back and their ears were twitching as if listening to whatever surprised them."

- "I thought Miro was surprised because It kind of jumped (rolled?) back and was twitching it's ears like it was trying to process what just happened or was scared."

- "Miro quickly scooted backwards and then turned its ears like it was surprised by something."

- "I thought Miro was surprised because he went back and had wide eyes."

- "The way he backs up quickly and wiggles his ears makes him look surprised."

- "I thought Miro was surprised because of the way he stepped back while moving his ears. It was as if he saw something that caught his attention."

Angry was mostly confused with "Annoyed" and "Excited". This was also due to similarities in movements in these expressions (for both "Annoyed" and "Excited"), as well as the colours shown on Miro (mostly for "Annoyed"). In this case, it is interesting that those who did not select "Angry" and selected "Annoyed" instead, left comments that reflected that Miro could have been angry (similar to what was observed for "Fearful"). Below we list some examples of the comments.

- "He seems agitated and moving back and forth. The red light also tells me so."

- "He's just sort of anxiously shuffling in place."

- "It looks like he's trying to get your attention in a confrontational way."

- "was backing up and going forward continually. Looked angry. Had a red light."
- "I thought Miro was annoyed because he made quick movements forward and backwards and his eyes were wide open.

- "It moved back and forth sort of jumpy like. I think this indicates it is not happy and wants to call your attention to that fact that it is annoyed."

- "It seems like the movements resemble happiness because of the front and back motion

- "Miro moved around like he couldn't stand still, like he was excited."

- "I think Miro is somewhat excited and happy here. Maybe a bit surprised because he's moving back and forth. In any case, he is certainly active."

Disgusted was mostly confused with "Fearful" and "Annoyed". This was mainly due to the similarities in movements, such as similarities in eye and body movements for these expressions. Examples of comments are given below.

- "I thought Miro was fearful and surprised because they kept opening their eyes and seeing something before closing them and backing away."

- "Miro seemed sad and scared because of the way its eyes looked when open and the fact that it closed its eyes and scooted backwards. It seemed like it was backing away from a threat or something."

- "I thought it was fear because it was moving backwards like a 'caution' and closing its eyes."

- "Miro seemed sad and scared because of the way its eyes looked when open and the fact that it closed its eyes and scooted backwards. It seemed like it was backing away from a threat or something." 
- "I thought Miro was angry or fearful because he was shaking his head in defiance or was closing his eyes and backing away from something he was afraid of. I guess another possibility would be that he was disgusted by something now that I think of it."

- "I thought Miro was annoyed because it was slowly backing away from the situation, but it didn't seem scared and instead was sort of moving its head downward and across its body in an almost angry fashion."

- "I thought Miro was annoyed because it kept backing away, closing its eyes, and shaking its head to the side as if to say 'no'."

Annoyed was mostly confused with "Surprised" and "Bored". This was also mainly due to the similarities in the movements for these expressions. Examples of comments are below. In most comments related to "Surprise" and "Bored", participants expressed the emotion as "Curious" and some indicated that they would have selected "Curious" if it was available.

- "The way he tilts his head so dramatically makes me think of someone who can not believe what they just heard, and are tilting their ear forward to make sure they heard correctly."

- "It was tilting it's head like people do when they are surprised or disgusted by something."

- "I thought Miro was surprised because he kept moving his head back and forth, as if he was trying to figure out something that was a surprise to him."

- "My initial thought was that Miro seems confused, like when real dogs cock their heads. Surprise was the closest thing to confusion that I could pick."

- "I thought he was surprised because he seemed to be tilting his head, trying to understand or hear something. He was also calm. I would have picked curious if that was an answer."

- "Not sure. I would have chosen "curious" or "alert" if they were available because its head moves to the side like a dog when it is confused."

- "Based on it's head position and it's tilting, I wouldn't say Miro is interested currently. At the same time the eyes aren't fully open to show interest so I'd say maybe it's bored and giving that head tilting look to show me they're bored."

Taking those comments into consideration, it appears that the confusion of labelling affective expressions can be attributed to the limitations in Miro's movements. For example, as we could not change Miro's facial expressions (except for its eyes/eyelids) and were not able to bend its ears, it was hard to distinguish between the affective expressions where

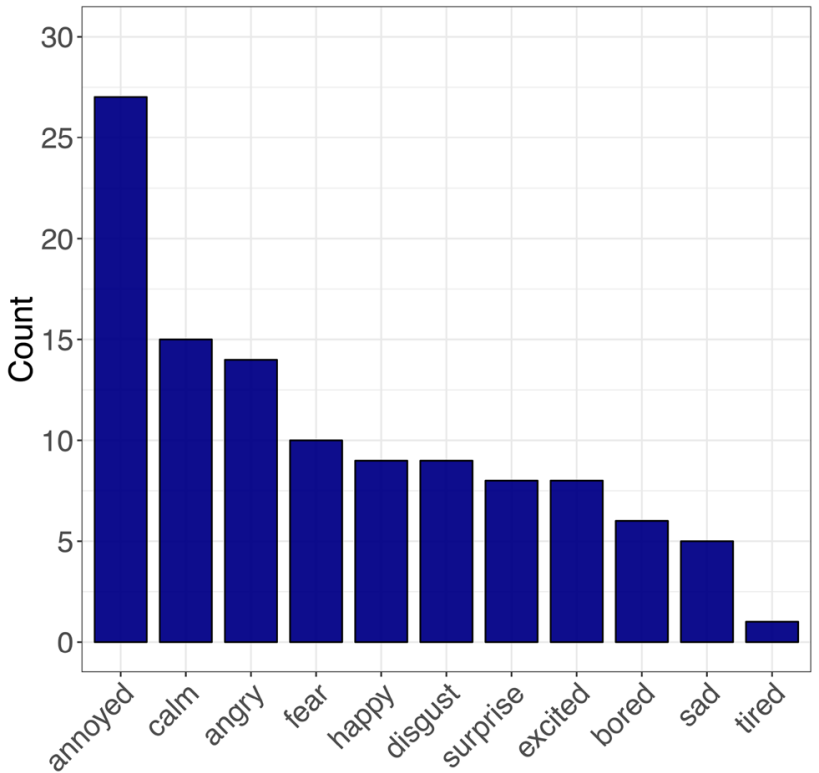

Fig. 4 Number of "not sure" selected for each emotion, reflecting uncertainty in deciding on a label for the robot's emotional expression

the eyes, the position of the head, or some body movements are very similar.

Furthermore, as the selection of "not sure" can indicate uncertainty, we analyzed how many times participants selected "not sure" (as mentioned above this could only be selected along with another choice). Figure 4 shows the results. We fit a linear mixed effects model to predict whether the selected affective expression was correct, based on whether the "not sure" option was selected. A random effect based on participant was fit. Selecting "not sure" was significantly and negatively correlated with selection of the correct answer ( $s e=0.050, t=-7.456, p<0.0001)$. In other words, those who did not select "not sure" (i.e., did not reflect uncertainty in their choice) recognized the 'correct' affective expressions (as they were designed) significantly more than those who selected "not sure".

\subsection{RQ2: Human and Animal Emotion Understanding}

Next, we asked how participants' ability of understanding human emotions affected their responses to Miro's expressions. If a participant is not capable of understanding human emotions very well, it is more likely that an incorrect answer can be due to a general difficulty in understanding affective expressions, as opposed to flaws in our design of the robot's affective expressions. Therefore, we analyzed how recognition of Miro's affective expressions changed based on participants' ability to recognize human emotions. Figure 5a shows the results. Recognition of the robot's affective expressions changed significantly and positively with partic- 
Fig. 5 Number of correct responses for Miro's expressions (max: 11) based on a the correct responses to the human images (max: 12), and b participants' reported difficulty in understanding emotions

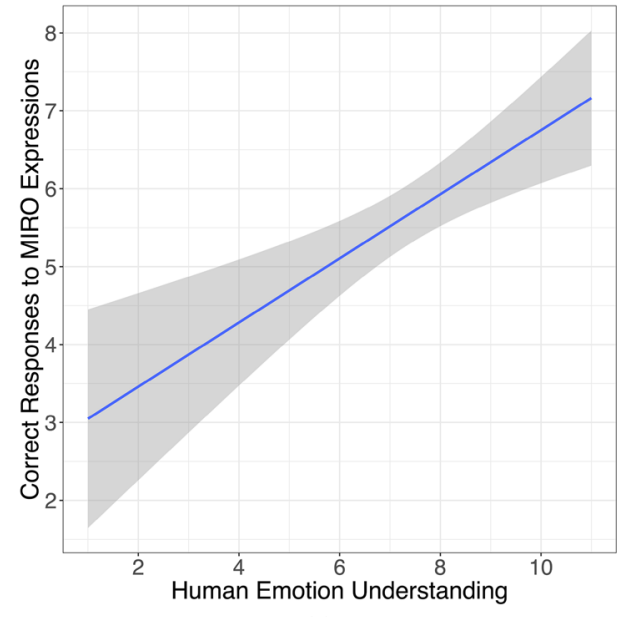

(a)

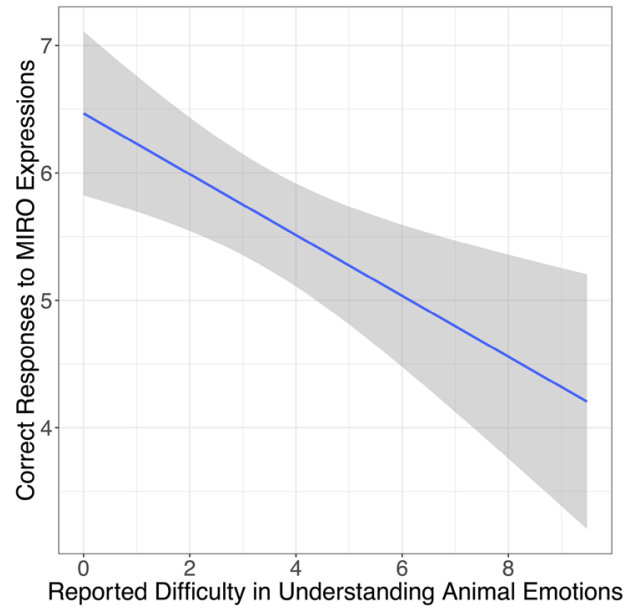

(b)
Table 3 Linear model predicting the number of correct answers to MIRO expressions based on participants' number of correct answers to human emotions (HumanUnd), perceived difficulty in understanding animal emotions (AnimalDiff), whether they have pets (hasPet), gender, and age

\begin{tabular}{lrrrl}
\hline Covariate & Estimate & $\mathrm{SE}$ & $\mathrm{t}$ & $\operatorname{Pr}(>|t|)$ \\
\hline Intercept & 3.073 & 1.184 & 2.595 & $<0.05$ \\
HumanUnd & 0.350 & 0.113 & 3.114 & $<0.005$ \\
AnimalDiff & -0.002 & 0.001 & -2.338 & $<0.05$ \\
Age & -0.011 & 0.019 & -0.571 & 0.569 \\
GenderMale & 0.305 & 0.416 & 0.732 & 0.466 \\
hasPet:TRUE & 0.950 & 0.623 & 1.525 & 0.130 \\
\hline
\end{tabular}

ipants' ability to recognize human emotions (as also shown in Table 3).

We further referred to participants' self-reported ability of understanding animal emotions as part of the animal exposure questionnaire. We asked if they have difficulty in understanding pets' emotions (as well as using a consistency check by asking if they had a good understanding of pets' emotions). As shown in Fig. 5b, those who reported that they have difficulty in recognition of animal emotions recognized Miro's expressions significantly less accurately (as also shown in Table 3) than those who reported little or no difficulty in understanding animal emotions.

To study significance of these results, a linear model was fit, predicting the number of correct responses to Miro's expressions based on the number of correct recognition of human emotions and participants' self-reported difficulty in understanding animal emotions. We also controlled for gender, age, and whether the participants had pets, as these three factors are shown to influence people's understanding of human emotions [30]. Results are shown in Table 3. Both human emotion recognition and self-reported difficulty in understanding animal emotions significantly affected

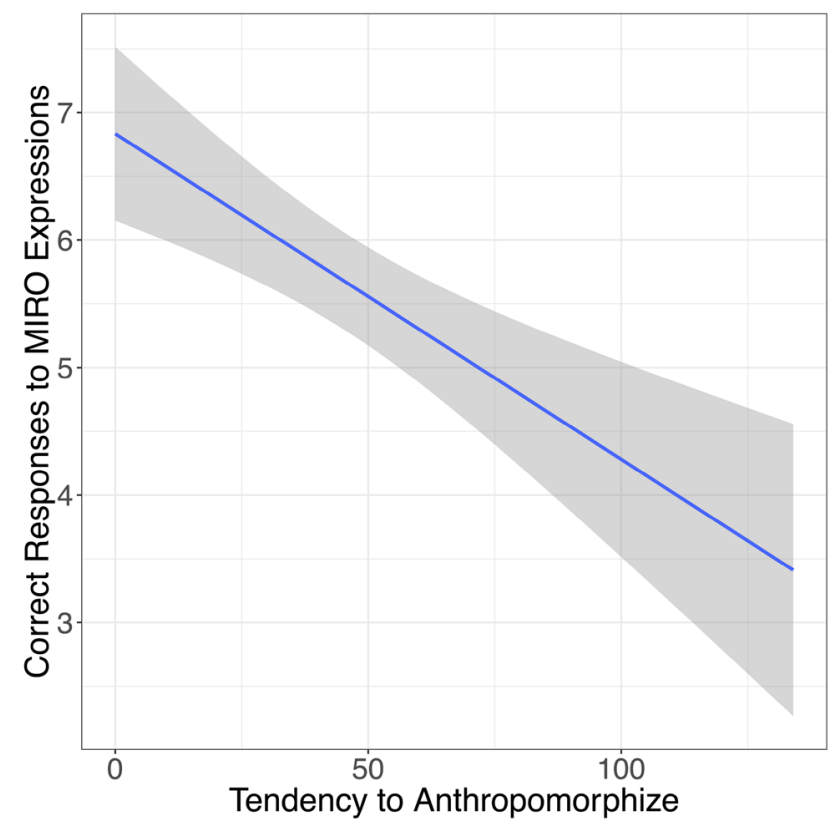

Fig. 6 Number of correctly recognized expressions based on participants' tendency to anthropomorphize

the number of correctly recognized affective expressions in Miro. We did not see any effect of gender, age, and whether participants had a pet on the recognition of Miro's emotions. These results confirmed our initial hypothesis regarding RQ2.

\subsection{RQ3: Tendency to Anthropomorphize}

It has been shown that the tendency to anthropomorphize can affect people's perception of computer agents, and can negatively affect their behaviour towards them (perhaps due to an uncanny valley effect) [52] which postulates that as agents become more and more human-like in appearance 
Fig. 7 a Number of correct responses to Miro's affective expressions (max: 11) based on whether the participants had/did not have a pet. $95 \%$ confidence intervals are visualized. The large confidence intervals for those who did not have a pet might be due to the limited number of participants in that group (13). b participants' response to whether they liked pets based on whether they had pets (which can act as a consistency check for the responses). This difference between the groups was significant $(z=3.330, p<0.001)$

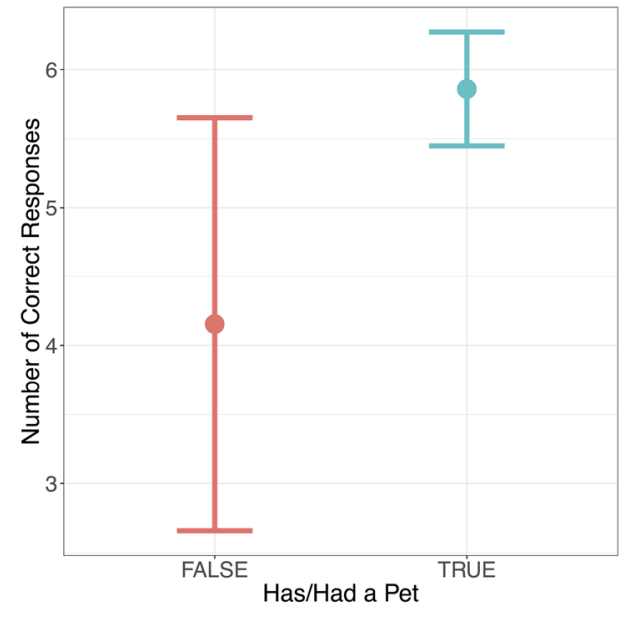

(a)

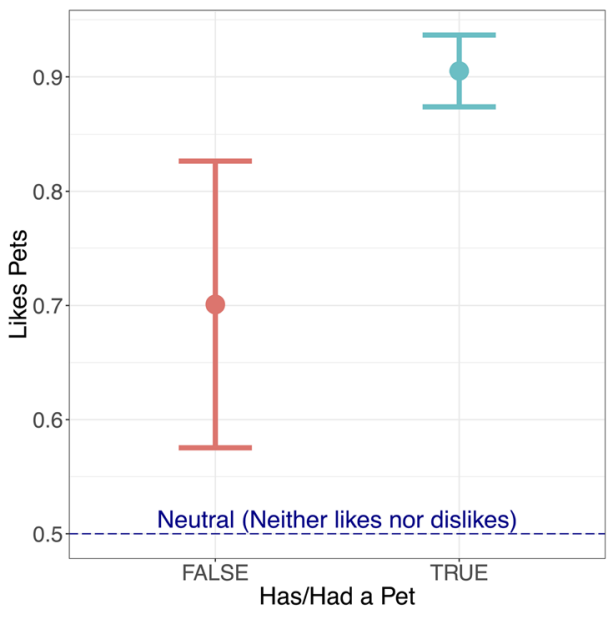

(b) and/or behavior, while still being clearly distinguishable from humans, they might reach a point where they are considered to be 'uncanny' or 'zombie-like', which triggers a response of repulsion in people [55]. Therefore, here we studied how the tendency to anthropomorphize-measured through the IDAQ questionnaire-affected participants' recognition of Miro's expressions.

Figure 6 shows the results. The general tendency to anthropomorphize significantly ( $p<.0001$ as measured through a linear model) and negatively affected recognition of Miro emotions, suggesting that those who attributed less human characteristics to Miro were more accurate in recognizing its expressions, thus supporting our hypothesis for RQ3.

\subsection{RQ4: Familiarity and Exposure to Animals}

Part of the animal exposure questionnaire concerned questions regarding pets and animals. A summery of the answers to those questions is presented below. While we did not find evidence for our initial expectation - that familiarity with animals/pets might increase recognition of Miro's affective expressions-we nevertheless report on the results for completeness purposes and in order to inform future research which might further investigate those factors.

- Question: Have participants ever had a pet (and if yes, what pet did they have)?: While the model shown in Table 3 suggests that having a pet is not a significant predictor for the recognition of Miro's affective expressions when the other effects are controlled for, the results suggested that those who had a pet had a significantly better recognition of Miro expressions. Results are shown in Fig. 7a. Further studies are needed to draw a meaningful conclusion on this difference, as we only had a very small sample size for those who did not have a pet (13 participants).
- Question: Do they have a close friend/family member who has a pet (and if yes, what pet do they have)?: 103 participants indicated that they had a friend who had a pet and only nine participants did not have a friend with a pet, four of which did not have a pet themselves either. We could not find an effect of having a friend with a pet on the recognition of Miro's affective expressions. This can be also due to the small number of participants who did not have a friend with a pet, and specifically those who neither had a pet, nor had a friend with a pet.

- Question: Do they like pets? The majority of the participants indicated that they liked pets. Rated on a continuous scale from 0 to 1 , the average score was $0.88\left(Q_{1}=\right.$ $0.85, Q_{3}=1$ ), with a median of 0.91 . As expected, the very few participants who indicated that they did not like pets were those who did not have pets, as shown in Fig. 7b. Due to this similarity in the responses, we could not observe any effect of how much participants liked pets on their recognition of the robot's affective expressions.

- Question: Are they scared of pets? Results for being scared of pets were consistent with those for "liking pets". Most participants indicated that they were not scared of pets. The average score for this category was 0.26 $\left(Q_{1}=0.04, Q_{3}=0.43\right)$ with a median of 0.17 . Similar to "liking pets", we did not observe any effect of how much people were scared of pets on their recognition of the robot's affective expressions.

- Question: Do they believe that animals can have emotions like humans? Most participants believed that animals can have emotions. As expected, those who had pets had a significantly stronger belief about this $(z=$ $2.378, p<.05)$. However, similar to the previews questions (i.e., questions 3 and 4), the responses were similar for most participants (mean: 0.84, median: 0.9, and $Q_{1}=0.7, Q_{3}=1$ ), therefore we did not observe any 


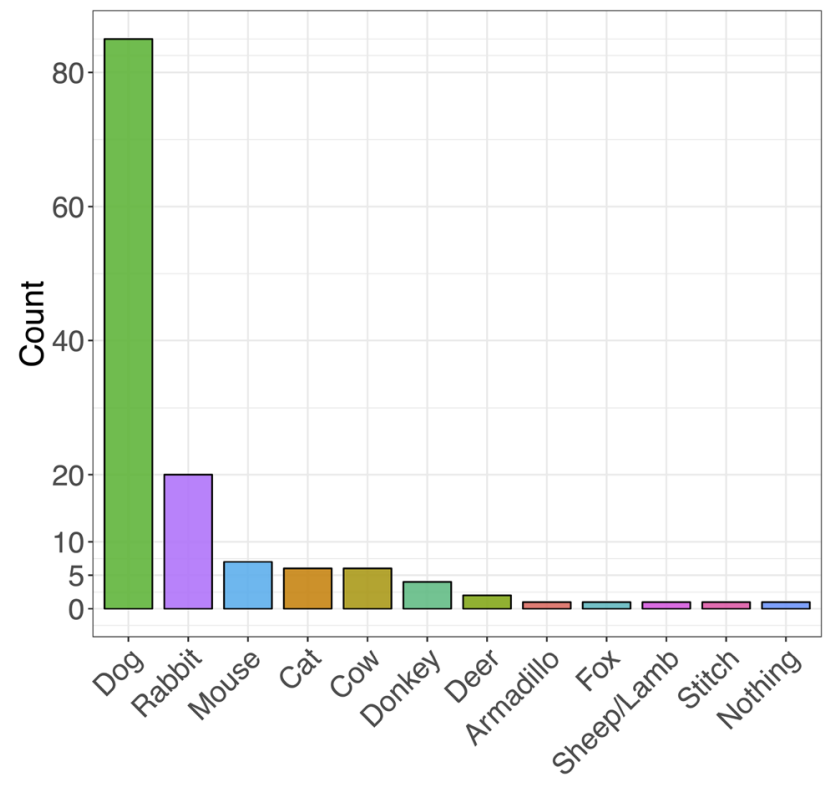

Fig. 8 Miro's associated animal, as reported by the participants

effect of this belief on the recognition of the robot's affective expressions.

The consistency in the responses to these questions indicates that the participants paid attention to the task and carefully answered all the questions.

\subsection{RQ5: General Perception of Miro}

To understand participants' general perception of Miro, we asked about Miro's gender and the animal participants associate with it. Figures 8 and 9 show the results.

"A rabbit in looks, a dog in personality": The majority of participants viewed Miro as a dog, followed by a rabbit (the two animals that are mostly associated to Miro in the related studies). One participant indicated that Miro was similar to a rabbit in looks and a dog in personality (perhaps due to being inspired by dog behaviour for its design). Interestingly, answers such as "Mouse", "Cat", and "Cow" were also repeated, suggesting that Miro can be perceived as a variety of different animals, beyond a dog and a rabbit. Many participants in our study reported that they have a dog or know someone who has a dog as a response to the type of pet in questions 1 and 2. However, since dogs are the most popular pets in Canada and the USA, it is not clear whether having a dog lead to perceiving Miro as a dog or not. As there are many responses categorizing Miro as a dog, as well as very few instances in the other categories, we could not conduct a meaningful analysis to study correlations between the pet that a participant had and the animal that was associated to Miro.

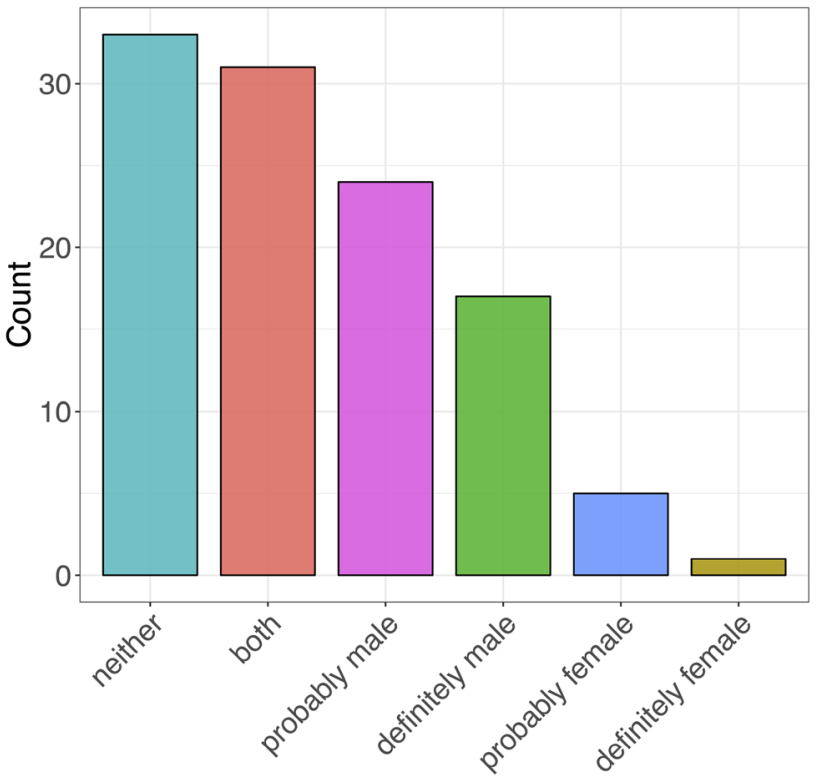

Fig. 9 Perception of Miro's gender, as reported by the participants

Furthermore, as shown in Fig. 9, Miro's gender was mostly seen as neutral, as the majority of the participants either chose "neither" or "both" for its gender.

Finally, we asked how Miro was perceived on humanlike, machine-like, and animal-like dimensions. While some human-like expressions were used for the design of the affective expressions, it is expected that it would not be perceived as human-like. Also, understanding how it is perceived on machine-like and animal-like dimensions is specifically informative for understanding the possibility of an uncanny valley effects in the future studies.

Results are shown in Fig. 10. Miro was perceived to be both animal-like and machine-like, while, as expected, it was

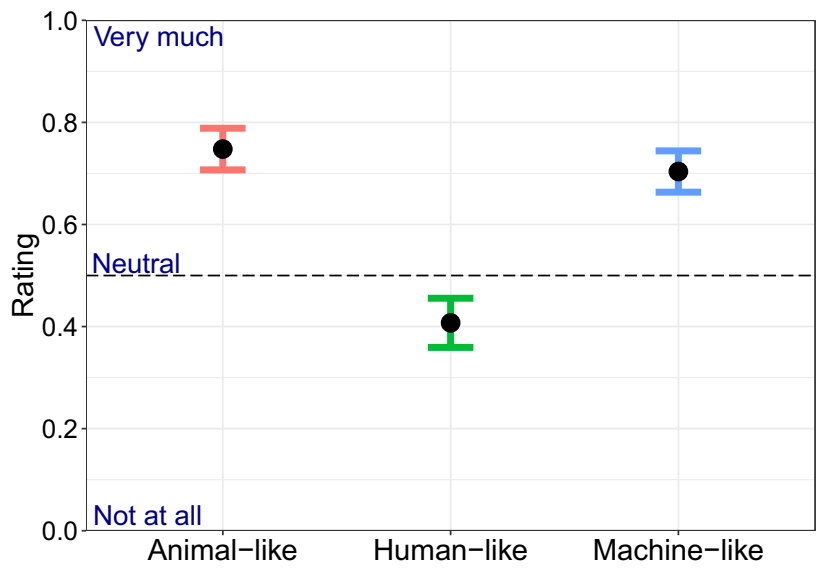

Fig. 10 Evaluation of Miro on human-like, machine-like, and animallike scales 
perceived to be significantly less human-like $(p<.0001$, measured through t-tests).

\section{Discussion}

The capability to perceive and express emotions is considered to be important for creating truly socially interactive robots [2] that can engage in meaningful and enjoyable social relationships with humans [56]. This capability is important in many domains (e.g., healthcare and education) and can be critical for adoption of socially interactive robots, engagement with people, and gaining users' trust, including users with specific cognitive disabilities such as dementia [57]. To move towards developing emotionally intelligent social robots, in this paper we proposed design guidelines and presented an evaluation of eleven affective expressions for the Miro robot. Miro's embodiment has deliberately been created to be animal-like, but not faithfully mimicking any particular animal specie. This raises the potential for those design guidelines to also be utilized and applied to other zoomorphic robots. We further asked how different aspects of Miro (e.g., its gender) are perceived by the participants, which would be informative for other human-robot interaction studies, specifically with the Miro robot.

To address the first research question (RQ1), the 11 designed affective expressions were evaluated through a video study. All expressions were recognized significantly more than the chance level, and except for fear and bored, the correct response was selected more than the other options. Furthermore, five expressions (i.e., happy, sad, surprised, excited, and tired) were recognized by half of the participants, and the correct response was selected significantly more than the other options for six of the 11 expressions in total. In cases where this difference was not significant, the correct option was still selected, but along with another option.

In a qualitative analysis of participants' comments about the expressions, we found that confusions of the expressions were mostly due to the robot's limited ability in showing facial expressions. Specifically, the expressions that were confused with each other have indeed similarities in eye and body movements (in both humans and animals), while the differences are mostly reflected through movements and positions of the ears, eyebrows, and mouth, all of which cannot be controlled in the Miro robot. In addition, participants' general ability to understand emotions-which was measured through a human emotion recognition task and a question about the difficulty of understanding animal emotions-affected the recognition of the robot's affective expressions (as hypothesized for RQ2). These two effects suggested that the lower accuracy in recognizing some of the affective expressions might be due to Miro's limited embod- iment, as well as people's general level of understanding of emotions, as opposed to shortcomings in the design of the affective expressions. For example, even in the robot Kismet where motors mimicking 'face muscles' could be controlled, surprise was mostly confused with fear [19]. Similarly, in our case, fear was mostly confused with surprise. The difficulty in distinguishing between fear and surprise is in fact supported by the literature on adults' and children's recognition of these emotions, and in most cases this challenge is attributed to people's limited attention to the position of the eyebrows, which is a key in distinguishing between these emotions [58] (and being an animal-like robot, Miro does not possess eyebrows).

Furthermore, adults [8] and children [7] are reported to have difficulty in distinguishing between disgust and anger, which are two of the expressions that did not receive a high accuracy in our study. Recognition of the affective expressions is also expected to be improved if the robot's expressions are observed in a specific context of humanrobot interaction rather than watching them in isolation and with no explicit contextual cues. Due to all of these factors, it remains challenging to understand whether and how it is possible to improve these affective expressions in Miro, and future work is needed to address it. However, it might be difficult to address all of those shortcomings in a zoomorphic design for a (relatively) small and inexpensive robot, so as a general lesson it might be recommended, in future work, to focus on those affective expressions in Miro which were recognized reliably.

In general, happiness and sadness are reported to be the fastest and, developmentally speaking, earliest recognized emotions [59,60]. Also, recognition of positive emotions are believed to be faster/earlier and more accurate than that of the negative emotions [59,61]. While our participants were all adults, in our study, too, recognition of positive emotions was more accurate than the negative emotions.

One interesting observation in our study was that the recognition of expressions was significantly affected by participants' tendency to anthropomorphize (to address RQ3), and those with a higher anthropomorphism tendency had more difficulty in recognizing the expressions (as hypothesized in RQ3). One explanation could be that a higher anthropomorphism tendency can lead to higher expectations for the robot to be accurate in its expressions, therefore affecting recognition of the expressions negatively.

Other than the effects of participants' anthropomorphism tendency and their understanding of animals' and humans' emotions, as discussed above, we investigated participants' attitude towards animals (i.e., if they had pets or knew someone who had pets, if they liked animals, if they were scared of animals, and if they believed that animals can have emotions like humans) to address RQ4. These factors could affect recognition of emotions, and if not considered, could lead to 
confounds when studying the other above mentioned effects (i.e., in RQ1 and RQ2). We did not find any effect of these factors on the recognition of Miro's affective expressions; however, consistency in participants' responses to these questions and the comparison between responses of pet owners and non-pet owners acted as an additional sanity and consistency check to ensure that the participants paid attention to the questions in the study.

Note that in this paper we only focused on Miro's affective expressions reflected through its body gestures and movements. Affective expressions can be improved by further focusing on lights in a similar manner as proposed in [11]. Further, adding sounds (e.g., like in [62]) can improve understanding of these expressions. However, here, we only focused on design of expressions as adding other modalities such as sound could have led to a confound for evaluating the effectiveness of Miro's face and body gestures. Improving these gestures would be especially beneficial in situations where light and sound are not effective, for example in well-lit rooms, in crowded/noisy/ large environments, or for people with a hearing problem.

Finally, studying participants' perception of Miro (to address RQ5) suggested that, while Miro is mostly associated with a dog or a rabbit, there is a large variety of other animals that were associated to Miro by different participants. Also, Miro was mostly perceived to be gender neutral, making it a suitable robot for human-robot studies, especially where the robot's gender might affect participants' behaviour or attitude towards the robot or trigger unhelpful expectations (e.g., see [53]). However, we expect that with minor changes in its appearance, researchers can still design it in a way that it represents a specific gender, or indeed personality, if needed, similar to the effects of personalization that have been documented for Roomba, a commercially available, machine-like, vaccuum cleaning robot [63]. Our results suggest that a gender effect might not be much of a concern in studies in human-robot interactions with Miro, which can be advantageous in many potential applications for Miro where gender and associated gender biases and stereotypes might play an important role. Research in robotics shows that often people readily attribute a specific gender to a robot, either as part of a personalization process, and even for a clearly machine-like robot [63], or because of the robot's humanoid shape [64]. This even applies to mass-produced humanoid robots which have originally been designed to be gender neutral [65], while still being implicitly considered as gendered in terms of voice and morphology [66].

Lastly, we know that perceiving robots and virtual agents as very human-like (but not completely) [67] may lead to disliking them and can negatively affect quality of humanrobot interactions [67]. Therefore, we asked how humanlike, animal-like, and machine-like participants perceived the robot to be (note that while the robot was not human-like in appearance, some of the designed affective behaviours mimicked human behaviour). Our results suggested that there might be less of a concern for an uncanny valley for the Miro robot, as it is perceived highly machine-like (while also being perceived very animal-like).

\section{Limitations and Future Work}

Our work had several limitations. First, as discussed earlier, there are multiple factors that could have affected recognition of Miro's expressions beyond the design decisions. Improving upon these expressions might necessitate a substantial redesign of the embodiment of a commercially available robot, which is typically not within the remit of HRI research teams (apart from possible minor changes, adaptations, and personalizations). Future work could combine sounds and contextual cues in combination with the behavioural cues we investigated in the present study. Also, the effect of using lights on the recognition of emotions can be investigated further in future work. Further, due to limitations in Miro's movements (e.g., its ears cannot be bent and facial expressions are limited to the eyelids), the designed behaviours do not fully mimic animals' behaviour. In addition, as we were not able to find standard questionnaires that assess humans' understanding of animal emotions, we used measures for the recognition of human emotions instead. One option we considered was using pictures of dogs (e.g., the ones used in [68]), to measure "dog emotion recognition", but as suggested by the same authors, humans' recognition of dogs' emotions is often not as straightforward based on photographs, because different parameters such as ear and tail movements can be very important. Development and validation of questionnaires to assess the affective expressions of animals, and specifically animal-like robots, could help in better understanding the accuracy of Miro's expressions, as well as how to improve them. Also, while we did not observe effects of participants' behaviours and attitudes towards animals on their recognition of Miro's expressions, this might have been due to having very few non-pet owners, those who disliked animals, or those who were scared of animals. Future studies with a different group of participants who do not own a pet, dislike animals, or are scared of animals can be informative, to see if these factors could affect participants' behaviour and attitude towards zoomorphic robots.

Finally, while an online video study may affect the results as compared to in person studies, where the participants interact directly with the Miro robot, crowdsourcing studies are a well established method in HCI and HRI studies, which are shown to have comparable outcomes as compared with the direct recruitment methods [69]. This approach has become even more common as a result of COVID-19. Finally, future work in applying these design guidelines for designing affec- 
tive expressions for other animal-like robots could lead to valuable comparative studies and further insights into the development of a future framework of affective expressions for zoomorphic robots.

\section{Conclusion}

As the capability of connecting with users on an affective level is becoming more and more important for the acceptance and success of interactions between humans and intelligent agents, this paper proposed design guidelines for the implementation of 11 affective expressions for a zoomorphic robot called Miro. The evaluation results were promising and suggested that many of the expressions can be recognized correctly, especially by those who have a good understanding of human emotions in general. While recognition of some expressions were not as high as the others, the results suggested that Miro's limitations and participants' general challenges in understanding emotions in both animals and humans could have resulted in a lower accuracy, as opposed to shortcomings in the proposed design guidelines. Other factors such as participants' anthropomorphism tendency, as well as their familiarity with and attitudes towards animals and pets were investigated, among which the anthropomorphism tendency was found to significantly and negatively affect the recognition of emotions.

We suggest that our guidelines for affective expressions can be generalized (to a reasonable extent) and help with implementing the affective expressions for other animal-like social robots. Our study also revealed how Miro is perceived in general, which can help with its selection for specific tasks, as well as understanding possible confounding effects (e.g., caused by perceived gender and type of animal) in humanrobot interaction studies with Miro. To summarize, we hope that our research provided a significant step towards enhancing affective expressivity of zoomorphic robots in general, and the Miro robot in particular, thus putting Miro and its fellow conspecifics on the path of becoming truly socially and emotionally intelligent robots.

Acknowledgements This research was funded by the Network for Aging Research at the University of Waterloo. This research was undertaken, in part, thanks to funding from the Canada 150 Research Chairs Program. We would like to thank Jesse Hoey and Chrystopher L. Nehaniv for their comments on this work, and Zhuofu Tao for his help with development of the platform for the online study.

Funding This study was funded by the Network for Aging Research at the University of Waterloo and the Canada 150 Research Chairs Program.

\section{Declarations}

Conflict of interest The authors declare that they have no conflict of interest.
Data availability The datasets generated during the current study are available from the corresponding author on reasonable request.

\section{References}

1. Dautenhahn K, Nehaniv CL, Walters ML, Robins B, Kose-Bagci H, Mirza NA, Blow M (2009) KASPAR-a minimally expressive humanoid robot for human-robot interaction research. Appl Bionics Biomech 6(3-4):369

2. Dautenhahn K (2007) Socially intelligent robots: dimensions of human-robot interaction. Philos Trans R Soc B Biol Sci 362(1480):679-704

3. O'Brien HL, Toms EG (2008) What is user engagement? A conceptual framework for defining user engagement with technology. J Am Soc Inf Sci Technol 59(6):938

4. Chowanda A, Flintham M, Blanchfield P, Valstar M (2016), Playing with social and emotional game companions. In: International conference on intelligent virtual agents, pp. 85-95. Springer

5. Ghafurian M, Budnarain N, Hoey J (2019) Role of emotions in perception of humanness of virtual agents. In: Proceedings of the 18th international conference on autonomous agents and multiagent systems (International Foundation for Autonomous Agents and Multiagent Systems, Richland, SC, 2019), AAMAS'19, pp 1979-1981

6. Lench H, Flores S, Bench S (2011) Discrete emotions predict changes in cognition, judgment, experience, behavior, and physiology: a meta-analysis of experimental emotion elicitations. Psychol Bull 137:834. https://doi.org/10.1037/a0024244

7. Widen SC, Russell JA (2010) The "disgust face" conveys anger to children. Emotion 10(4):455

8. Widen SC, Russell JA, Brooks A (2004) Anger and disgust: discrete or overlapping categories. In: 2004 APS annual convention. Boston College, Chicago

9. Collins EC, Prescott TJ, Mitchinson B, Conran S (2015),MIRO: a versatile biomimetic edutainment robot. In: Proceedings of the 12 th international conference on advances in computer entertainment technology, pp 1-4

10. Mitchinson B, Prescott TJ (2016) MIRO: a robot "Mammal" with a biomimetic brain-based control system. In: Conference on biomimetic and biohybrid systems, pp 179-191. Springer

11. Collins EC, Prescott TJ, Mitchinson B (2015) Saying it with light: a pilot study of affective communication using the MIRO robot. In: Conference on biomimetic and biohybrid systems, pp 243-255. Springer

12. Becker-Asano C, Prendinger H, Ishizuka M, Wachsmuth I (2005) In: Proceedings of the international conference on active media technology (AMT 2005)

13. Poggi I, Pelachaud C, de Rosis F, Carofiglio V, De Carolis B (2005) Empathy for max (preliminary project report). In: Multimodal intelligent information presentation, pp 3-25. Springer

14. Velásquez JD (1999) An emotion-based approach to robotics. In: Proceedings 1999 IEEE/RSJ international conference on intelligent robots and systems. Human and environment friendly robots with high intelligence and emotional quotients (Cat. No. 99CH36289), vol 1, pp. 235-240. IEEE

15. Sosnowski S, Bittermann A, Kuhnlenz K, Buss M (2006) Design and evaluation of emotion-display EDDIE. In: 2006 IEEE/RSJ international conference on intelligent robots and systems, pp 3113-3118. IEEE

16. Dautenhahn K, Bond AH, Canamero L, Edmonds B (2002) Playing the emotion game with Feelix: What can a LEGO robot tell us about emotion?, vol 3. Springer 
17. Numata T, Asa Y, Kitagaki T, Hashimoto T, Karasawa K (2019) Young and elderly users' emotion recognition of dynamically formed expressions made by a non-human virtual agent. In: Proceedings of the 7 th international conference on human-agent interaction, pp 253-255

18. Saldien J, Goris K, Vanderborght B, Vanderfaeillie J, Lefeber D (2010) Expressing emotions with the social robot probo. Int J Soc Robot 2(4):377

19. Breazeal C (2003) Emotion and sociable humanoid robots. Int J Hum Comput Stud 59(1-2):119

20. Korcsok B, Konok V, Persa G, Faragó T, Niitsuma M, Miklósi Á, Korondi P, Baranyi P, Gácsi M (2018) Emotion and sociable humanoid robots. Front Psychol 9:1191

21. Gácsi Márta, Kis Anna, Faragó Tamás, Janiak Mariusz, Muszyński Robert, Miklósi Ádám (2016) Humans attribute emotions to a robot that shows simple behavioural patterns borrowed from dog behaviour. Comput Hum Behav 59:411-419

22. Löffler D, Schmidt N, Tscharn R (2018) Multimodal expression of artificial emotion in social robots using color, motion and sound. In: Proceedings of the 2018 ACM/IEEE international conference on human-robot interaction, pp 334-343

23. Häring M, Bee N, André E (2011) Creation and evaluation of emotion expression with body movement, sound and eye color for humanoid robots. In: 2011 RO-MAN, pp 204-209. IEEE

24. Bethel CL, Murphy RR (2007) Survey of non-facial/non-verbal affective expressions for appearance-constrained robots. IEEE Trans Syst Man Cybern Part C Appl Rev 38(1):83

25. Lakatos G, Gácsi M, Konok V, Bruder I, Bereczky B, Korondi P, Miklosi A (2014) Emotion attribution to a non-humanoid robot in different social situations. PLoS One 9(12):114207

26. Morris PH, Doe C, Godsell E (2008) Secondary emotions in nonprimate species? Behavioural reports and subjective claims by animal owners. Cogn Emot 22(1):3

27. Konok V, Nagy K, Miklósi Á (2015) How do humans represent the emotions of dogs? The resemblance between the human representation of the canine and the human affective space. Appl Anim Behav Sci 162:37

28. Miklósi Á, Korondi P, Matellán V, Gácsi M (2017) Ethorobotics: a new approach to human-robot relationship. Front Psychol 8:958

29. Melson GF, Kahn PH, Beck AM, Friedman B, Roberts T, Garrett E (2005) Robots as dogs? Children's interactions with the robotic dog AIBO and a live Australian Shepherd. In: CHI-05 extended abstracts on human factors in computing systems, CHI EA'05, pp 1649-1652. Association for Computing Machinery, New York. https://doi.org/10.1145/1056808.1056988

30. Dawson L, Niel L, Cheal J, Mason G (2019) Humans can identify cats affective states from subtle facial expressions, UFAW

31. Wan M, Bolger N, Champagne FA (2012) Human perception of fear in dogs varies according to experience with dogs. PLoS One 7(12):51775

32. Maréchal L, Levy X, Meints K, Majolo B (2017) Experiencebased human perception of facial expressions in Barbary macaques (Macaca sylvanus). PeerJ 5:E3413

33. Nicastro N, Owren MJ (2003) Classification of domestic cat (Felis catus) vocalizations by Naive and experienced human listeners. J Comp Psychol 117(1):44

34. Pongrácz P, Molnár C, Miklósi Á (2006) Acoustic parameters of dog barks carry emotional information for humans. Appl Anim Behav Sci 100(3):228. https://doi.org/10.1016/j.applanim.2005. 12.004

35. Tami G, Gallagher A (2009) Description of the behaviour of domestic $\operatorname{dog}$ (Canis familiaris) by experienced and inexperienced people. Appl Anim Behav Sci 120(3-4):159

36. Demirbas YS, Ozturk H, Emre B, Kockaya M, Ozvardar T, Scott A (2016) Adultsability to interpret canine body language during a dog-child interaction. Anthrozoös 29(4):581
37. Canamero L (2001) Building emotional artifacts in social worlds: challenges and perspectives, emotional and intelligent II: the tangled knot of social cognition. AAAI Technical Report FS-01-02

38. Ghafurian M, Lakatos G, Tao Z, Dautenhahn K (2020) Design and evaluation of affective expressions of a zoomorphic robot. In: International conference on social robotics, pp 1-12. Springer

39. Topál J, Miklósi Á, Csányi V, Dóka A (1998) Attachment behavior in dogs (Canis familiaris): a new application of Ainsworth's (1969) Strange Situation Test. J Comp Psychol 112(3):219

40. Klausz B, Kis A, Persa E, Miklósi Á, Gácsi M (2014) A quick assessment tool for human-directed aggression in pet dogs. Aggress Behav 40(2): 178

41. Vas J, Topál J, Gácsi M, Miklósi A, Csányi V (2005) A friend or an enemy? Dogs reaction to an unfamiliar person showing behavioural cues of threat and friendliness at different times. Appl Anim Behav Sci 94(1-2):99

42. Konok V, Dóka A, Miklósi Á (2011) The behavior of the domestic $\operatorname{dog}$ (Canis familiaris) during separation from and reunion with the owner: a questionnaire and an experimental study. Appl Anim Behav Sci 135(4):300

43. Hampshire V, Robertson S (2015) Using the facial grimace scale to evaluate rabbit wellness in post-procedural monitoring. Lab Anim 44(7):259

44. Matsumiya LC, Sorge RE, Sotocinal SG, Tabaka JM, Wieskopf JS, Zaloum A, King OD, Mogil JS (2012) Using the Mouse Grimace Scale to reevaluate the efficacy of postoperative analgesics in laboratory mice. J Am Assoc Lab Anim Sci 51(1):42

45. Quaranta A, Siniscalchi M, Vallortigara G (2007) Asymmetric tailwagging responses by dogs to different emotive stimuli. Curr Biol 17(6):R199

46. Hasegawa M, Ohtani N, Ohta M (2014) Dogs body language relevant to learning achievement. Animals 4(1):45

47. Keating SC, Thomas AA, Flecknell PA, Leach MC (2012) Evaluation of EMLA cream for preventing pain during tattooing of rabbits: changes in physiological, behavioural and facial expression responses. PLoS One 7(9):e44437

48. Burn CC (2017) Bestial boredom: a biological perspective on animal boredom and suggestions for its scientific investigation. Anim Behav 130:141

49. Reisenzein R, Bördgen S, Holtbernd T, Matz D (2006) Evidence for strong dissociation between emotion and facial displays: the case of surprise. J Personal Soc Psychol 91(2):295

50. Terwogt MM, Hoeksma JB (1995) Colors and emotions: preferences and combinations. J Gen Psychol 122(1):5

51. Nijdam NA (2009) Mapping emotion to color, Book Mapping emotion to color, pp 2-9. www.semanticscholar.org/paper/Mapping -emotion-to-color-Nijdam/5f0de6e7bc1d5443243f9f42f2379db9 639a933d

52. Ghafurian M, Budnarain N, Hoey J (2021) Improving humanness of virtual agents and users' cooperation through emotions. IEEE Trans Affect Comput. https://doi.org/10.1109/TAFFC.2021.3096831

53. Siegel M, Breazeal C, Norton MI (2009) Persuasive robotics: the influence of robot gender on human behavior. In: 2009 IEEE/RSJ international conference on intelligent robots and systems, pp 2563-2568. IEEE

54. Waytz A, Cacioppo J, Epley N (2010) Who sees human? The stability and importance of individual differences in anthropomorphism. Perspect Psychol Sci 5(3):219

55. MacDorman KF, Ishiguro H (2006) The uncanny advantage of using androids in social and cognitive science research. Interact Stud 7(2):297

56. Dautenhahn K (1995) Getting to know each other artificial social intelligence for autonomous robots. Robot Auton Syst 16(2-4):333

57. König A, Francis LE, Joshi J, Robillard JM, Hoey J (2017) Qualitative study of affective identities in dementia patients for the design 
of cognitive assistive technologies. J Rehabil Assist Technol Eng 4:1-5. https://doi.org/10.1177/2055668316685038

58. Roy-Charland A, Perron M, Young C, Boulard J, Chamberland JA (2015) The confusion of fear and surprise: a developmental study of the perceptual-attentional limitation hypothesis using eye movements. J Genet Psychol 176(5):281

59. Camras LA, Allison K (1985) Children's understanding of emotional facial expressions and verbal labels. J Nonverbal Behav 9(2):84

60. Saarni C (1999) The development of emotional competence. Guilford Press

61. De Sonneville L, Verschoor C, Njiokiktjien C, Op het Veld V, Toorenaar N, Vranken M (2002) Facial identity and facial emotions: speed, accuracy, and processing strategies in children and adults. J Clin Exp Neuropsychol 24(2):200

62. Ritschel H, Aslan I, Mertes S, Seiderer A, André E (2019) Personalized synthesis of intentional and emotional non-verbal sounds for social robots. In: 2019 8th International conference on affective computing and intelligent interaction (ACII), pp 1-7

63. Sung J, Grinter RE, Christensen HI (2009) Pimp My Roomba: designing for personalization. In: Proceedings of the SIGCHI conference on human factors in computing systems, CHI'09, pp 193-196. Association for Computing Machinery, New York. https://doi.org/10.1145/1518701.1518732

64. Søraa RA (2017) Mechanical genders: How do humans gender robots? Gender. Technol Dev 21(1-2):99. https://doi.org/10.1080/ 09718524.2017.1385320

65. Pandey AK, Gelin R (2018) A mass-produced sociable humanoid robot: pepper: the first machine of its kind. IEEE Robot Autom Mag 25(3):40

66. Jackson RB, Williams T, Smith N (2020) Exploring the role of gender in perceptions of robotic noncompliance. In: Proceedings of the $2020 \mathrm{ACM} / \mathrm{IEEE}$ international conference on human-robot interaction, HRI'20, pp 559-567. Association for Computing Machinery, New York. https://doi.org/10.1145/3319502.3374831

67. Mathur Maya B, Reichling David B (2016) Navigating a social world with robot partners: a quantitative cartography of the Uncanny Valley. Cognition 146:22-32

68. Bloom T, Friedman H (2013) Classifying dogs (Canis familiaris) facial expressions from photographs. Behav Process 96:1

69. Bartneck C, Duenser A, Moltchanova E, Zawieska K (2015) Comparing the similarity of responses received from studies in Amazon mechanical turk to studies conducted online and with direct recruitment. PLoS One 10(4):1
Publisher's Note Springer Nature remains neutral with regard to jurisdictional claims in published maps and institutional affiliations.

Moojan Ghafurian is a Research Assistant Professor at the Department of Electrical and Computer Engineering at the University of Waterloo. She got her PhD from the Pennsylvania State University and was the Inaugural Wes Graham postdoctoral fellow from 2018-2020 at David R. Cheriton School of Computer Science at the University of Waterloo. Her research interests and background are in humancomputer/robot interaction, social robotics, affective computing, and cognitive science. Her research explores computational models of how humans interact with intelligent agents to inform user-centered design of emotionally intelligent agents in multiple domains.

Gabriella Lakatos is a Lecturer at the School of Engineering and Computer Science and a member of the Adaptive Systems Research Group at the University of Hertfordshire, UK. She completed her $\mathrm{PhD}$ in the field of Ethology at the Eotvos Lorand University, Hungary, analysing human-dog Interactions, after which she transferred her skills to the fields of Ethorobotics and Human-Robot Interactions (HRI), while participating in several largescale cross-disciplinary EU projects. Her expertise and research interests lie in social robotics and HRI, including biologically inspired behaviour design, social credibility, trust and user acceptance of assistive and companion robots. She is also guest editor of a special issue on trust, acceptance and social cues for the Paladyn, Journal of Behavioral Robotics.

Kerstin Dautenhahn, IEEE Fellow, is full Professor and Canada 150 Research Chair in Intelligent Robotics at University of Waterloo in Ontario, Canada. She has a joint appointment with the Departments of Electrical and Computer Engineering and Systems Design Engineering and is cross-appointed with the David R. Cheriton School of Computer Science at University of Waterloo. She is Visiting Professor at the University of Hertfordshire, UK. In Waterloo she is director of the Social and Intelligent Robotics Research Laboratory (SIRRL). She frequently gives invited keynote talks at international conference and has senior editorial roles with international journals (including Editor in Chief of the journal Interaction Studies). 